\title{
Research on Key Parameters of Boost Pressure Limiting Valve
}

\author{
Shang $\mathrm{HaO}^{1}$, and Guang-ming $\mathrm{Li}^{2^{*}}$ \\ ${ }^{1}$ School of Information Engineering, Wuhan University of Technology, Wuhan, China, 430070 \\ ${ }^{2}$ ANYANG XINSHENG MACHINE TOOL CO, LTD., Anyang, China, 455000 \\ ${ }^{*}$ Corresponding author
}

\section{Key Words: Air Gap Distance ,Suction Distance, Cut-off Point}

\begin{abstract}
In this paper, according to the internal structure of the boost pressure limiting valve, the actual working characteristics of the boost pressure limiting valve is analyzed, and the key factors affecting the work curve and cut-off point of the boost pressure limiting valve are summarized, then experiments with different size of the suction distance and the air gap distance between moving core and static core by loading the PWM control signal, we can see that the different size of the air gap distances and suction distances correspond to the different pressure curves and cut-off points from the experimental results.
\end{abstract}

\section{Exhaust Gas Turbo Charge System Working Principle}

The exhaust turbo charging system utilizes the energy of the exhausted gas from the automobile engine to drive the turbine by controlling the flow of the exhaust gas, and the turbine in the turbine chamber rotates the impeller of the intake chamber, then, the impeller presses the air sent by the air cleaner pipe into the pressurized cylinder to improve the power of the engine. Compared with traditional naturally aspirated engines, exhaust turbo charged engine has higher torque engine power, more fully burning of fuel combustion to reduce harmful emissions, and saves more energy. The turbo charging system mainly consists of turbine, impeller, actuator, boost pressure limiting valve, exhaust bypass valve .When the supercharging pressure is too high, the exhaust bypass valve is opened and the exhaust gas is directly discharged into the trachea through the bypass passage to adjust the supercharging pressure not to be excessively high. Exhaust bypass valve opened or not is controlled by the actuator and the actuator is dominated by the boost pressure limiting valve which is directly controlled by the automobile engine control module ECU. [1]

\section{Boost Pressure Limiting Valve}

The boost pressure limiting valve is equivalent to a proportional three-way solenoid valve connected to the booster compressor outlet, the boost pressure regulator and the low pressure inlet (compressor inlet) through a rubber hose. The boost pressure limiting valve can control the force applied to the exhaust bypass valve in the exhaust turbo charging system by controlling the opening of the exhaust bypass valve and to control the intake pressure of the turbine chamber indirectly.

The boost pressure limiting valve controlled by the engine control unit through way of the duty cycle mode power supply when the engine is in high speed and large load conditions, can communicate low pressure ventilation with another terminal, so that the pressure applied to the diaphragm of the supercharging pressure adjusting unit is lowered, and the opening degree of the exhaust bypass valve is reduced, while the supercharging pressure is increased. The pressure sensor provides feedback to the ECU to provide closed feedback loop control, which adjusts the pulse width by the ECU to make precise control under any operating conditions to ensure that the exhaust bypass valve target pressure and boost pressure, $[2,3]$ boost pressure limiting valve internal structure is shown in Figure.1. 


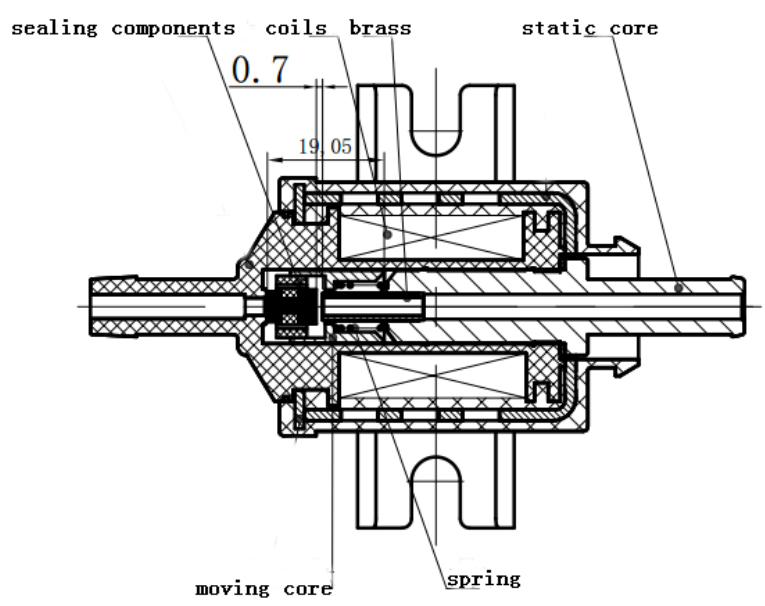

Figure.1Internal structure of valve

It can be seen from Figure 1, the boost pressure limiting valve mainly consists of the moving core, static core, sealing components, springs, brass and enameled coils, and its working principle is through the PWM signal changes to control the opening time ratio of intake port $\mathrm{P}$ and exhaust port $\mathrm{T}$ to achieve the pressure adjustment indirectly. When the coil is energized, the inner moving core and the static iron core of the coil become magnets and overcome the spring force to attract each other, then, port $\mathrm{P}$ is blocked, while port A and port Tare directly connected, this period is the pressure of port A adjustment process; When the power is off, the moving core and the static core of the coil are separated due to the spring elastic support. Then, the port $\mathrm{T}$ is blocked and the port $\mathrm{A}$ is connected with the port $\mathrm{P}$. At this period, the pressure of port A is stable. Working process of the valve is shown in Figure. 2.

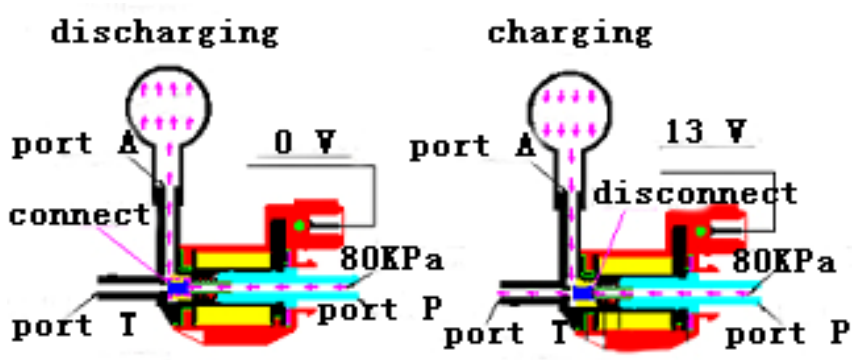

$$
\text { P--int ake I--exhaust A--control }
$$

Figure.2Direction of gas flow

When the signal is high, the entire coil due to the magnetic effect of the current magnetic field, moving iron core and static core will be magnetized to form a magnet. Magnetic field strength $\mathrm{H}$ and magnetic induction $\mathrm{B}$ have the following relationship:

$$
\mathrm{B}=\mu \mathrm{H}
$$

$\mu$ represents magnetic medium permeability, According to the law of Ampere Circle:

$$
\oint_{l} \mathrm{H} \cdot \mathrm{dl}=\sum \mathrm{Ni}
$$

If you do not consider the magnetic flux leakage and other parts of the air gap, only consider the armature of the trip, then: 


$$
\mathrm{NI}=\frac{\mathrm{l}_{0} \mathrm{~B}}{\mu_{0}}+\frac{\mathrm{l}_{1} \mathrm{~B}}{\mu_{1}}
$$

Where $\mathrm{N}$ is the turns number of the coil, I is the current through the wire, is the air gap distance in the coil, $\mu_{0}=4 \pi \cdot 10^{-7}$ is the air permeability, $\mathrm{l}_{1}$ is the soft iron length, $\mu_{1}=3 \cdot 10^{-3}$ is the soft iron ermeability.

Because $\mu_{0}$ is much smaller than $\mu_{1}$, the magnetic induction of the magnetic field can be approximated as:

$$
\mathrm{B}=\frac{\mathrm{N} \cdot \mathrm{I}}{\mathrm{l}_{0}} \mu_{0}
$$

Electromagnets with direct current solenoids, according to the formula to calculate the steady-state work of the electromagnet suction is[4]:

$$
F=\frac{\Phi^{2}}{2 S \mu_{0}}=\frac{B^{2}}{2 \mu_{0}} S
$$

In the formula (5), Фis the working air gap flux, Sis the cross-sectional area of the magnetic circuit, substituting formula (4) into formula (5) can obtain:

$$
\mathrm{F}=\frac{\mathrm{N}^{2} \mathrm{I}^{2} \mu_{0}}{2 \mathrm{l}_{0}^{2}} \mathrm{~S}
$$

It is can be seen from formula (6), the magnitude of the electromagnetic force is inversely proportional to the square of the air gap length, so, if you want to improve the solenoid coil suction, you can increase the number of coil turns or reduce the static core and moving core air gap when the PWM control signal in the same circumstances.

\section{Dynamic Analysis of Moving Core}

In the boost pressure limiting valve working cycle, when the access voltage $13.5 \mathrm{~V}$, the moving core will be instantly blocked intake port $\mathrm{P}$, while exhaust port $\mathrm{T}$ instantly be opened(The actual time may be smaller, because with the movement of the armature, the decreasing of air gap will cause a sharp increase in magnetic attraction, so, the suction time will be shorter).As the spring in the cavity of the moving distance is small, the spring force $F_{1}$ is constant forceapproximately, and the known spring mass is $98 \mathrm{~g}$, moving core mass $m_{2}$ is $3 \mathrm{~g}$, the instantaneous acceleration $a$ of moving iron core in the working state is:

$$
\mathrm{a}=\frac{\mathrm{F}-\mathrm{F}_{1}}{\mathrm{~m}_{2}}
$$

The magnetic forceF in the working voltage can be calculated for the size of 3.92Naccording to the relevant parameters, and the spring forceis $0.98 \mathrm{~N}$.The initial accelerationaof moving core is calculated as $0.98 \cdot 10^{3} \mathrm{~m} / \mathrm{s}^{2}$ on the basis offormula (7), then, the stroke of moving iron core is $0.5 \mathrm{mmrequires} 1 \mathrm{~ms}$. So, the moving core is blocked intake port $\mathrm{P}$ instantly, exhaust port $\mathrm{T}$ mouth is opened when the access voltage $13.5 \mathrm{~V}$ during the work cycle. The acceleration of the moving core is $0.32 \cdot 10^{3} \mathrm{~m} / \mathrm{s}^{2}$ during the low period of the PWM signal,and moving iron core and static core will not separate immediately because of spring bounce due to hysteresis effect. If the coil degaussing time is less than the PWM signal low-time maintenance time, then the moving core and static core will continue to pick-up, and said boost pressure limiting valve work in the cut-off point. 
Through the dynamic analysis of moving core stroke, it can be seen that changing the minimum suction distance of the boost pressure limiting valve can adjust the working cut-off point position and pressure of port $\mathrm{A}$, and choosing the proper suction distance can make the supercharging pressure limiting valve work in The best condition, realizes the A mouth gas pressure stability and the precise control.

\section{Key Parameters}

We can see that the main parameters to improve the working performance of the supercharging pressure limiting valve are the air gap distance and the minimum pull distance according to the working principle of the boost pressure limiting valve and dynamic analysis of moving iron core stroke,the key parameters are shown in Figure.3.

The electromagnetic force increases when the air gap distancel ${ }_{0}$ decreases, if the duty cycle is small, the armature will be able to move, with the duty cycle pressure curve shift to left, as shown inFigure.4, port $\mathrm{T}$ is opened in advance. But for the closure of port $\mathrm{P}$, because the minimum distance to close the $\mathrm{P}$ port has not changed, while closing time has not changed andthe time of opening $\mathrm{T}$ port will be longer. So the low duty cycle offset is more obvious, while the high duty cycle is not.

For the boost pressure limiting valve which works in the cut-off point, if reducing the suction distanced $_{0}$, it will increase the moving core from the magnetic field of electromagnetic force, while the cut-off point shift to left with the duty cycle pressure curve shift to left .

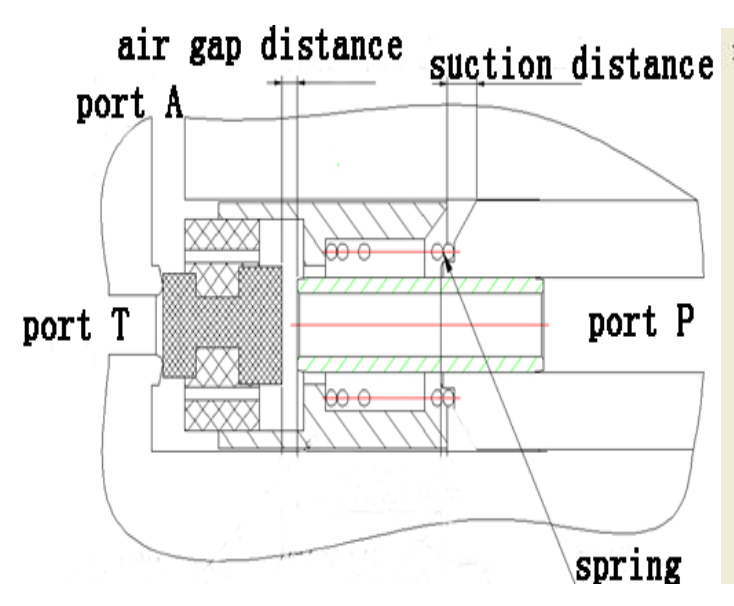

Figure.3Key parameter diagram

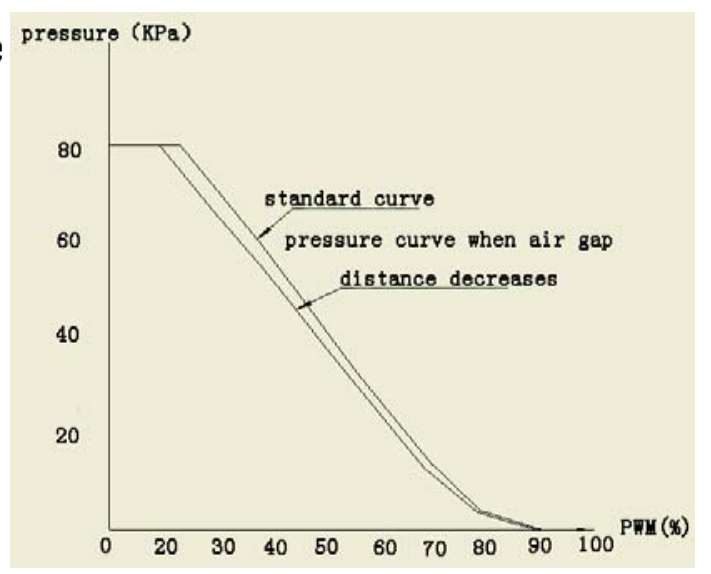

Figure.4Pressure / duty cycle curve

\section{Experimental Results}

Boost pressure limiting valve test system can be divided into vacuum regulator source, test control module and data processing module into three parts: Vacuum regulator module mainly supplies the entire test system with air stablepressure about $80 \mathrm{KPa}$ of the gas source (test medium); Test control module is designed to providethe driving power, data acquisition, control circuit, control procedures of the boost pressure limiting valve, and the upper, lower machine data transmission and reception between the communication protocol; the uppermachine is mainly responsible for the realization of data reception, processing, analysis and storage functions.

The air gap distance and the pull-in distance are taken as variables in the experiment. We have experiment(1) with different air gap distance about $0.64 \mathrm{~m} 、 0.73 \mathrm{~mm}$, and the control module take $5 \%$ $-100 \%, 100 \%-5 \%$ high $13.5 \mathrm{~V}$ PWM square wave as the driving signal, while samples the real - time pressure of port A, the experimental results are shown in Table 1.

As can be seen from Table 1, under the same duty cycle PWM control signal, $0.64 \mathrm{~mm}$ air gap distance from the measured pressure are less than $0.73 \mathrm{~mm}$ air gap distance corresponding to the measured pressure value, which corresponds to the left shift of the curve in Figure.4. It is proved that the working performance of boost pressure limit valve can be adjusted by changing the air gap distance. 
Table 1Duty cycle / pressure experimental data

\begin{tabular}{cccccccc}
\hline $\begin{array}{c}\text { Air gap } \\
\text { distance }\end{array}$ & \multicolumn{7}{c}{ Duty cycle/pressure actual test value } \\
\cline { 2 - 7 } $\boldsymbol{l}_{\mathbf{0}}^{(\mathrm{mm})}$ & $20 \%$ & $30 \%$ & $40 \%$ & $50 \%$ & $60 \%$ & $70 \%$ & PWM \\
0.64 & 67.39 & 46.09 & 27.88 & 14.74 & 6.19 & 0 & PWM increase \\
& 68.04 & 46.19 & 28.29 & 15.28 & 6.69 & 1.94 & PWM decrease \\
0.73 & 70.26 & 51.25 & 32.82 & 18.36 & 8.52 & 3.14 & PWM increase \\
& 71.52 & 52.2 & 34.44 & 19.53 & 9.2 & 3.65 & PWM decrease \\
\hline
\end{tabular}

We also have experiment(2) with different suction distance about $0.22 \mathrm{~m} 、 0.30 \mathrm{~mm}$, and the control module take 5\%-100\%, 100\% -5\% high 13.5V PWM square wave as the driving signal, while samples the real - time pressure of port A, the experimental results are shown in Figure.5and Figure.6.

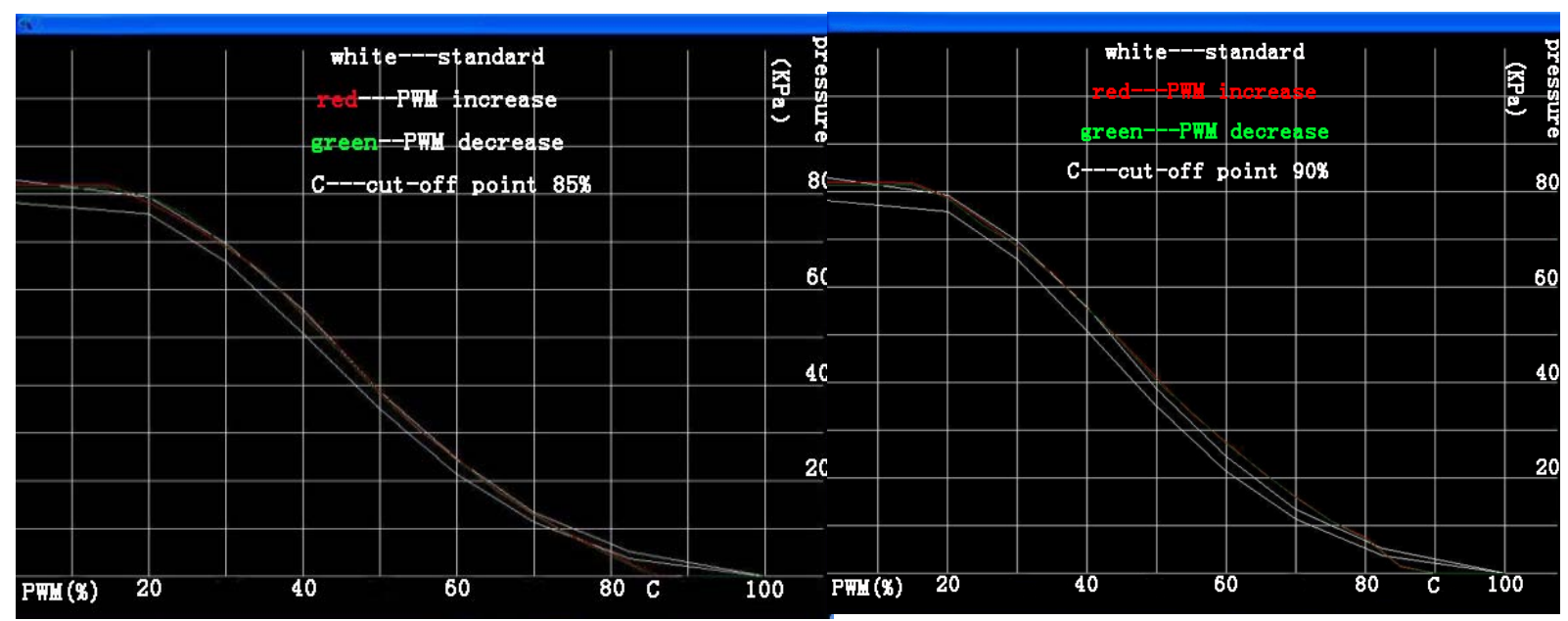

Figure.50.22 $\mathrm{mm}$ suction distance $\quad$ Figure.6 0.30mm suction distance

It can be seen from Figure. 5 and Figure.6, the cut-off points of the boost pressure limit valve with $0.22 \mathrm{~mm}$ and $0.3 \mathrm{~mm}$ suction distancerespectively are $85 \%$ and $90 \%$ PWM,but the duty cycle pressure curve is almost the same. It is verified that the cut-off point can be adjusted by changing the suction distance.

\section{Conclusions}

We can get the following conclusions through the analysis of the working principle and related parameters of the boost pressure limiting valve and the verification based on the results of the final experiments:

(1) The air gap distance between moving core and static core of boost pressure limiting valvedetermines the size of the working electromagnetic force.Reducing or increasing the air gap distance can be adjusted exhaust turbocharger system turbine chamber pressure to the appropriate range, and improve the stability of the turbocharger system stability.

(2) The suction distance between moving iron core and the static core of the boost pressure limiting valvehas an important impact on the cut-off point; precise control of the cut-off point of the boost pressure limiting valve plays an important role in the start-up time of the turbocharger.

\section{References}

[1] Gang Fu. Analysis of Advantages and Disadvantages of Exhaust Turbocharger [J]. Agricultural Machineryof HunanProvince,2013.7(7):109-110.

[2] Wei Wei, Ming-hai Li. Application of Turbocharging Technology in Automobile Engine [J]. China High - Tech Enterprises, 2010,(25): 22-24. 
[3] Wen-ting Chen, Ben-gang Lei, Shuang Liu, Ying-qin Hu, Fei Liu. Research on Noise Improvement of Exhaust Gas Bypass Valve of Turbocharger [J]. Proceedings of China Society of Automotive Engineers Annual Meeting, 2014:1441-1444..

[4] Lu-liang Lou, Hai-zhou Wang. Engineering Calculation Method of Electromagnetic Force in Solenoid Valve Design [J]. Missile and space vehicle technology, 2007, (1): 40-45. 